\title{
Clinical Efficacy of Plasma-Exchange in Patients with Progressive forms of Multiple Sclerosis and NMO-Spectrum Disease
}

Petrou P, Ben-Hur T, Vaknin-Dembinsky A, Abramsky O, Karussis D*

Multiple sclerosis Center and Unit of Neuroimmunology, Department of Neurology and Agnes Ginges center, Hadassah-Hebrew University Hospital

\begin{abstract}
Background: Plasma-exchange/plasmapheresis (PLEX) is an efficient treatment for several immune mediated diseases. In addition to its known efficacy in myasthenia gravis and Gulliain Barre syndrome, it has been also shown to be effective in certain patients with MS and other CNS demyelinating disorders, during an acute/sub-acute deterioration of the disease.
\end{abstract}

Aims and methods: We report the results of an open prospective study with PLEX in 36 patients with progressive forms of multiple sclerosis (either secondary progressive or relapsing-progressive) and 12 patients with NMO-spectrum disease. All patients had experienced a significant clinical deterioration in the year prior to inclusion (0.5-1 degree or more, in the EDSS scale or a severe relapse from which they did not fully recover) and responded partially or not at all to steroidal treatment. The mean EDSS score at inclusion was $5.91 \pm 1.46$. The mean EDSS for the MS subgroup was $5.95 \pm 1.3$ and for the NMO subgroup, $5.6 \pm 1.4$. The mean duration of the disease was $11.4 \pm 7.8$ years $(12 \pm$ 7.6 for the MS and 5.754 .59 for the NMO). All patients were treated with 5 courses of PLEX in 2 weeks, followed by a monthly course for one year.

Results: Twenty eight of the 48 patients $(58.3 \%)$ improved significantly in the EDSS score at year one post initiation of PLEX. The mean EDSS score declined from $5.91 \pm 1.46$ at inclusion, to $5.41 \pm 1.8$ at year one. This improvement was more pronounced in the NMO group: Ten out of twelve patients with NMO (83\%) improved and their mean EDSS score was reduced from $5.6 \pm 1.4$ before the treatment to $4.7 \pm 1.5$ EDSS score post PLEX. In the whole group there were 16 patients with over imposed relapses (relapsing-progressive course) with a total of 26 relapses in the year prior to the inclusion; the number of relapses during the year following PLEX was reduced from 26 to 4 . In general patients with prominent myelitic involvement had the most impressive response to the treatment. Five patients suffered from minor infections and one was admitted with sepsis. No other major side effects were observed.

Conclusion: PLEX may benefit some patients with progressive MS and NMO and thus may represent an alternative second line treatment modality for such patients with highly active disease, especially those with myelitic forms, and recent deterioration that did not respond to steroids. Larger, controlled studies are warranted to confirm the efficacy of PLEX in these subgroups of MS.

Keywords: Plasmapheresis; Therapeutic plasma exchange; GulliainBarre syndrome; Immunoglobulins; NMO-spectrum disease

\section{Introduction}

Plasmapheresis, also known as therapeutic plasma exchange (PLEX), is a procedure that separates the blood components, exchanging the plasma (typically with donor plasma or albumin), and returning the other components, primarily red blood cells, to the patient. PLEX has been proven to be effective in various autoimmune diseases, including neurological ones, such as Gulliain-Barre syndrome, idiopathic thrombocytopenic purpura and myasthenia gravis $[1,2]$.

Since the late 1980s PLEX has been tried in several studies on MS patients, but with inconsistent effects. This may be related to: a. the small size of the studies, b. the lack of homogeneity in the treatment porotocols, c. the use of PLEX as an adjuvant therapy to other immunomodulatory modalities and $\mathrm{d}$. the patient populations and type/course of MS, which greatly varied among these studies.

In two initial small size studies in 1985 patients with chronic MS were treated with PLEX as an add-on therapy to cyclophosphamide, intramuscular immunoglobulins or steroids and azathioprine $[3,4]$. The first one showed that significantly more patients in the PLEXgroups stabilized or improved at 5 months, as compared with the sham group. The second, (in which PLEX was applied with steroids and azathioprine) showed only a transient improvement in 7 out of the 10 PLEX-treated patients.

A subsequent double blind study including 116 patients, compared the clinical efficacy of PLEX (11 courses in a period of 11 weeks) with that of sham treatment for acute attacks of MS, in combination with cyclophosphamide and ACTH [5]. A moderately enhanced improvement (not statistically significant) was observed in the PLEXgroup after 2 weeks. The improvement was mainly evident in the RRMS group and not in the progressive patients.

In a larger and better organized trial, the Canadian Co-operative Study, 168 patients, who had deteriorated $\geq 1$ degree on the EDSS scale during the previous year, were treated with iv cyclophosphamide and daily oral prednisone $(n=55)$, or with alternate day oral prednisone and daily cyclophosphamide plus weekly PLEX for 20 weeks $(n=57)$ 56 untreated patients serving as the control group [6]. At 6 months, the percentage of treatment failure in each group was: $35 \%$ in the cyclophosphamide group, $32 \%$ in the PLEX group and $29 \%$ in the placebo group. These differences were not statistically significant.

*Corresponding author: Karussis D, Department of Neurology, Hadassah University Hospital, Ein-Kerem, IL91120, Jerusalem, Israel, Tel: 97226776939; E-mail: dimitrios@hadassah.org.il

Received June 29, 2016; Accepted July 23, 2016; Published July 31, 2016

Citation: Petrou P, Ben-Hur T, Vaknin-Dembinsky A, Abramsky O, Karussis D (2016) Clinical Efficacy of Plasma-Exchange in Patients with Progressive forms of Multiple Sclerosis and NMO-Spectrum Disease. J Mult Scler (Foster City) 3:181. doi:10.4172/2376-0389.1000181

Copyright: (C) 2016 Petrou P, et al. This is an open-access article distributed under the terms of the Creative Commons Attribution License, which permits unrestricted use, distribution, and reproduction in any medium, provided the original author and source are credited. 
In a more recent and randomized trial, which represented the "revival" of PLEX as a treatment modality for MS, PLEX (7 courses within 14 days) was for the first time checked as a single modality, in patients with various types of acute CNS demyelinating diseases (including ADEM and neuromyelitic types of MS, either chronic or relapsing) [7]. All included patients suffered acute episodes of neurological deterioration which did not improve with iv steroids. Clinical improvement was noted in $8 / 19(42,1 \%)$ of the PLEX-treated patients compared with $1 / 17(5.9 \%)$ in the sham group. When the patients were switched from PLEX to sham and from sham to PLEX, the clinical beneficial effects of PLEX noted in the first part of the trial, were strengthened, in favor of PLEX.

A follow up study by Keegan et al. [8] suggested that patients with myelitis and Pattern II immunopathogenesis (according to Luccineti et al., a predominantly antibody-mediated disease) had the best response to PLEX. None of the responders belonged to the type-I (classical MS) and type-III according to the histopathological classification of the disease [9]. Similar results were obtained in an additional small trial in patients with severe attacks of inflammatory demyelinating CNS diseases. The improvement was mainly evident during the treatment and faded off later.

A more recent retrospective analysis of 153 patients treated with PLEX for a steroid-refractory CNS demyelinating episode, showed that 59\% exhibited moderate to marked functional neurological improvement within 6 months following treatment. Plasma exchange was less effective for patients with multiple sclerosis who subsequently developed a progressive disease course. Radiographic features associated with a beneficial PLEX response were the presence of ringenhancing lesions and/or mass effect [10].

Logically, since in neuromyelitis optica (NMO) the immunopathology is closer to that of type-II histopathological phenotype of MS, NMO patients are expected to benefit from PLEX. Indeed two small open studies and a restrospective one in NMO patients during an acute attack of the disease, showed an early and significant improvement following PLEX in most of the treated patients [11-13]. An AAN task committee upon evaluating the neurological indications of PLEX gave a level B recommendation for its use as second-line treatment of steroid-resistant exacerbations in relapsing forms of MS and of NMO [14].

On the other hand, PLEX was rather ineffective in most of the studies in chronic progressive or secondary progressive MS [3,4]. In purely progressive MS, a study of combination treatment with azathioprine and PLEX in 8 patients with SPMS led to the conclusion that PLEX did not improve clinical outcomes or prevent the formation of Gd-EDPA enhancing lesins in MRI [15]. However, the total MS lesion load was significantly lower and central motor conduction times decreased significantly during PLEX treatment.

We report here the results of an open prospective study with PLEX in 36 patients with progressive forms of multiple sclerosis (either secondary progressive - SPMS- or relapsing progressive - RPMS-) and 12 patients with NMO-spectrum disease, who all had a significant clinical deterioration in the year prior to inclusion (0.5-1 degree in the EDSS scale or a severe relapse from which they did not fully recover) and responded partially or not at all to intensive steroidal treatment. All patients were treated (induction) with 5 courses of PLEX in 2 weeks, followed by monthly sessions for one year.

\section{Methods}

Thirty six patients with progressive forms of MS and 12 with NMO- spectrum disease, all with significant clinical deterioration during the year prior to the inclusion, who did not respond (or responded only partially) to high dose steroidal treatment, were included in this open prospective study.

\section{Inclusion criteria}

1. Definite diagnosis of MS according to Poser's criteria or definite diagnosis of NMO

2. Secondary progressive or relapsing progressive course of disease

3. Deterioration of at least one degree in the EDSS scale (if EDSS lower than 5.5) or 0.5 degree in EDSS (when baseline EDSS $>5.0$ ) during the year preceding their inclusion to our study, or had at least one major relapse with incomplete recovery.

4. Deterioration despite the chronic use of at least one immunomodulatory treatment

5. Failure to respond (or partial response) to steroids during the recent clinical deterioration

\section{Exclusion criteria}

1. The presence of other systemic autoimmune diseases, excluding thyroiditis

2. The presence of significant other systemic diseases

3. The presence of active infection

4. History of thrombosis or hypercoagulable state

The mean age of the included patients was $42.2 \pm 13.8$, mean duration of the disease was $11.4 \pm 7.8$ years and the mean EDSS score at inclusion, $5.91 \pm 1.46$. The mean age for the subgroup of MS was $46.2 \pm$ 11.3 and for the subgroup of NMO $32.1 \pm 11.2$. The mean EDSS for the MS subgroup was $5.95 \pm 1.3$ and for the NMO subgroup was $5.6 \pm 1.4$. The mean duration of the disease was $11.4 \pm 7.8$ years. (12 \pm 7.6 for the MS and $5.75 \pm 4.59$ for the NMO) (Table 1). Eight out of the 12 NMOspectrum patients were positive for serum anti aquaporin- 4 antibodies.

Nineteen patients have been previously treated with interferon, 11 with azathiorpine, 3 with Glatiramer acetate, 6 with mitoxantrone and 10 with natalizumab, all with insufficient response to those treatments (the clinical deterioration occurred despite the chronic use of at least one of the above treatments, during the year preceding the inclusion). Patients were treated with 5 courses of PLEX within two weeks followed by monthly treatments for 12 months and neurological status (EDSS) and number of relapses were evaluated after one year.

\section{Results}

\section{Clinical effects}

In total, 28 of the 48 patients (58\%) improved significantly in the EDSS score. The mean EDSS score declined from $5.91 \pm 1.46$ to $5.41 \pm 1.8$. This improvement was more pronounced in the NMO group: Ten out of twelve patients with NMO (83\%) improved and their mean EDSS score was reduced from $5.6 \pm 1.4$ before the treatment to $4.7 \pm 1.5$ (Table 2 and Figure 1).

\begin{tabular}{|c|c|c|c|}
\hline & Total $\mathbf{n = 4 8}$ & MS $\mathbf{n}=\mathbf{3 6}$ & NMO $\mathbf{n = 1 2}$ \\
\hline Age & $42.2 \pm 13.7$ & $46.2 \pm 11.3$ & $32.1 \pm 11.2$ \\
\hline Disease duration & $11.4 \pm 7.8$ & $12 \pm 7.6$ & $5.75 \pm 4.59$ \\
\hline EDSS before PLEX & $5.91 \pm 1.46$ & $5.95 \pm 1.3$ & $5.6 \pm 1.4$ \\
\hline $\begin{array}{c}\text { Predominant myelitic } \\
\text { involvement }\end{array}$ & 30 & 18 & 12 \\
\hline
\end{tabular}

Table 1: Demographic data of our patients. 
In the whole group, 46 out of the 48 included patients $(95.8 \%)$ remained progression free, during the year following PLEX; $58.3 \%$ improved in the EDSS score, $37.5 \%$ remained unchanged (but stopped progressing) and only $4.2 \%$ ( 2 patients) continued to deteriorate (Table 2A). In the NMO group the percentages of improvement, stabilization and deterioration were even more significant: $83.3 \%, 16.7 \%$ and $0 \%$, respectively); in the group of RP MS, the improvement rate was $71.4 \%$. In the subgroup with secondary progressive form of MS, the improvement rate was lower $(43.3 \%)$; the percentage of stabilized patients in this subgroup was $53.3 \%$. These clinical effects are summarized in Table 2. In general patients with myelitis had the most significant response to the treatment.

In the whole group there were 16 patients with (over imposed) relapses (relapsing-progressive course) with a total of 26 relapses (16 in the RP group and 10 in the NMOS group) in the year prior to the inclusion; the number of relapses during the year following PLEX, was reduced from 26 to 4 ( 2 in the RP group and 2 in the NMOS group) (Figure 2).

\section{Safety and adverse events}

Five patients suffered from minor infections and one was admitted with sepsis. No other major side effects were observed. Minor adverse effects included fatigue on the day following PLEX, or local infections at the place of the administration of the iv catheter.

\section{Discussion}

In this open prospective study we evaluated the clinical efficacy of PLEX, given chronically for one year, in progressive forms of MS and in NMO. Our data show beneficial clinical effects of the treatment in this selected group of progressive patients with very active disease who deteriorated significantly during the year prior to the inclusion. The clinical benefits were more pronounced in the NMO subgroup and in

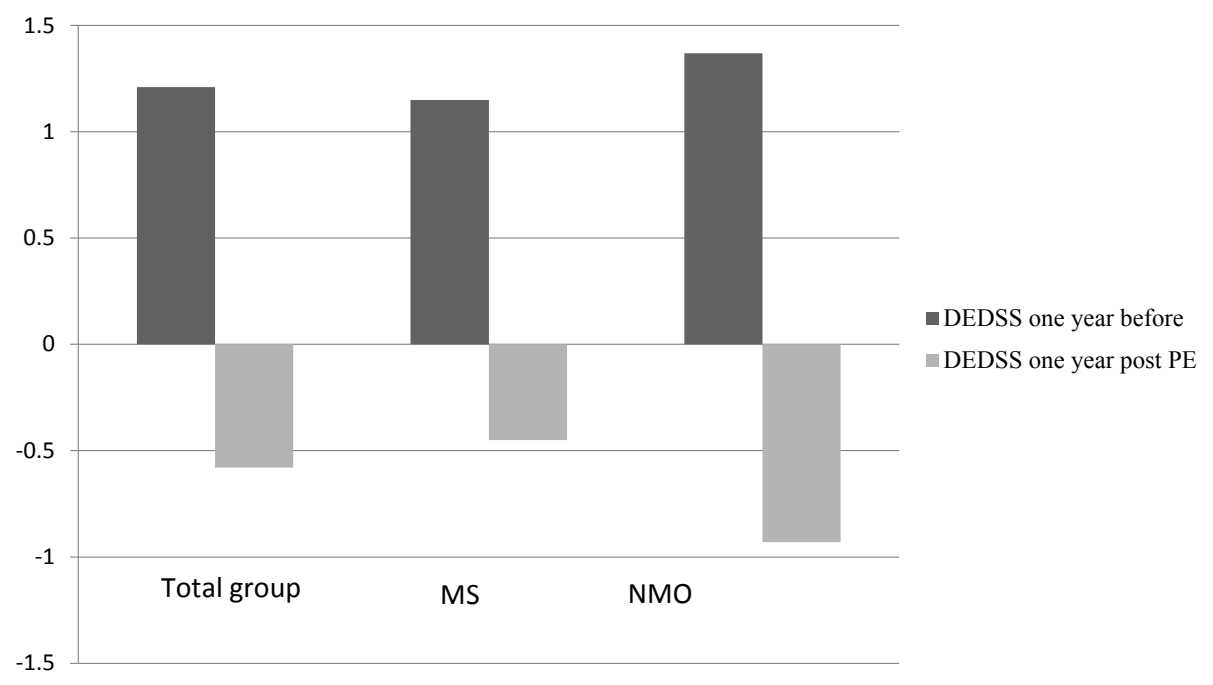

Figure 1: EDSS change in the year before inclusion vs. the year of treatment with PLEX.

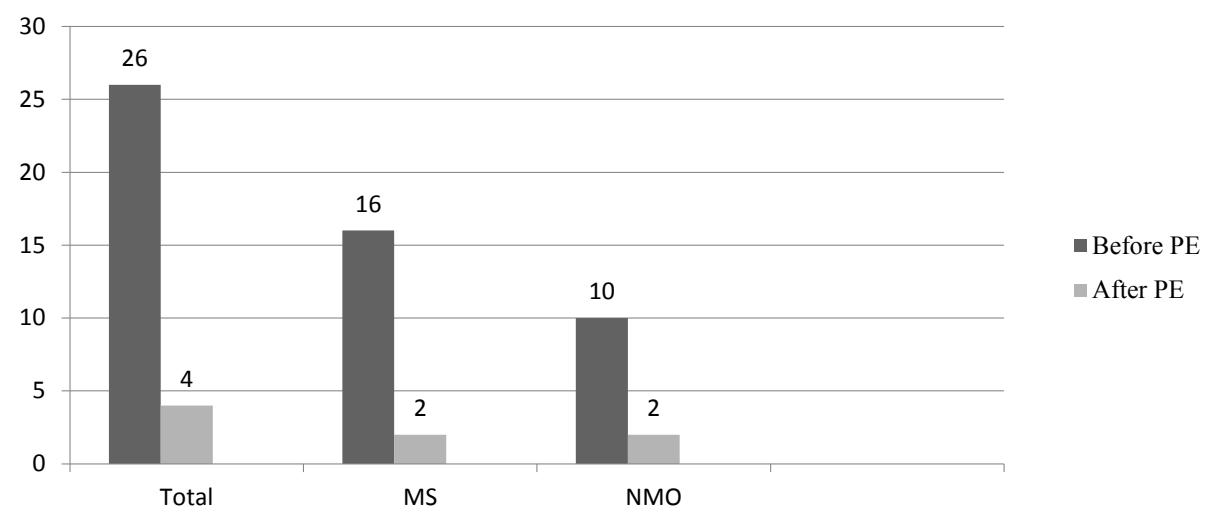

Figure 2: Number of relapses during the year before inclusion and in the year of treatment with PLEX.

\begin{tabular}{|c|c|c|c|}
\hline & EDSS at inclusion & EDSS at one year post PLEX & $\begin{array}{c}\text { EDDS progression during the } \\
\text { year before the inclusion }\end{array}$ \\
\hline DEDDS at one year post-PLEX \\
\hline RP MS $(n=18)$ & $5.91 \pm 1.46$ & $5.41 \pm 1.8$ & $+1.2 \pm 1.14$ \\
\hline SP MS $(n=18)$ & $5.91 \pm 1.2$ & $5.79 \pm 1.7$ & $+0.6 \pm 0.4$ \\
\hline NMO $(n=12)$ & $6.51 \pm 0.9$ & $6.16 \pm 0.8$ & $-0.45 \pm 0.6$ \\
\hline
\end{tabular}

Table 2A: Changes in the EDSS score in the subgroups of PLEX-treated patients. 


\begin{tabular}{|c|c|c|c|}
\hline & Improved & Stable & Deteriorated \\
\hline Total & 28 & 18 & 2 \\
\hline RP MS & 5 & 0 & 1 \\
\hline SP MS & 13 & 16 & 1 \\
\hline NMO & 10 & 2 & 0 \\
\hline
\end{tabular}

Table 2B: Change in clinical status following PLEX, according to the EDSS scale.

patients with myelitic involvement. 95\% of the whole group of patients remained progression free during the year following PLEX and $85 \%$ were relapse-free (Figure 1).

Previous clinical trials testing the efficacy of PLEX in MS have shown variable, though generally positive results and beneficial clinical effects $[4,6,7,14,16-20]$. The MS populations in these studies were not similar and the treatment protocols greatly varied. In many of these trials PLEX was not used as a sole treatment modality but as an add-on treatment, which complicates the interpretation of the results.

In the trial from Mayo Clinic, the patients who mainly benefited from the treatment were those with myelitis in whom a predominantly humoral, antibody mediated mechanism seems to be involved in the pathogenesis of their CNS demyelinating disease [8].

More recently, additional open retrospective trials showed that plasma-exchange was effective is acute relapses of MS, in severe optic neuritis, in relapses of NMO, as rescue therapy in clinically isolated syndromes and acute worsening of MS and in pediatric demyelinating syndromes [21-25]. An MRI-based study showed that in patients with steroid-refractory deterioration of MS, plasma exchange induced a partial of complete resolution of the active lesions in the MRI in $80 \%$ of the treated patients [26].

The rationale behind the use of plasma exchange in MS comes from various recent data depicting the crucial role of B cells and autoantibodies in the pathogenesis of the disease and especially in its progression. B cells have traditionally been considered to play a secondary T-cell-dependent role, producing antibodies that may promote tissue destruction by recruiting macrophages and through activation of the complement pathway [27]. Additionally, activated B cells can act as antigen-specific APCs for T cells and produce costimulatory molecules that influence the differentiation of T cells from Th1 to Th2 cells [28]. Patients with MS have increased B-cell numbers in the CNS, mainly memory cells and short-lived plasmablasts [29]. Plasmablasts persist in the cerebrospinal fluid (CSF) throughout the course of MS, and the numbers of these cells correlate with intrathecal immunoglobulin G (IgG) synthesis (oligoclonal antibodies, one of the hallmarks of MS diagnosis) and with active inflammatory disease [29,30]. Moreover, B cells, plasma cells, autoantibodies, and complement have been detected in MS lesions [31,32], indicating their implication in demyelination. Additional indications for antibody-mediated mechanisms in MS come from the presence of ectopic lymphoid follicles in the CNS of patients with MS [29,33-35], especially those with progressive disease. It appears, therefore, that as the disease evolves into the progressive stage, the inflammation becomes compartmentalized and predominantly mediated by B cells. Such "slow" in situ inflammation can be detected even in the cortex and the deep grey matter and seems to correlate with the progression of disability $[33,35-50]$.

However, one may logically question the mechanism of action of a treatment such as PLEX that acts peripherally and "filters" the antibodies from the blood, on such central mechanism of localized/ compartmentized inflammation in progressive MS, especially since antibodies are large molecules with limited trafficking through the blood brain barrier (BBB). However, although antibodies do not pass through an intact $\mathrm{BBB}$, their trafficking may be possible when this barrier is inflamed and damaged as in the case of active CNS disease [51]. This may provide a possible explanation of the efficacy of PLEX in our study, which included patients with active disease and recent deterioration (ie with a compromised BBB). In support of this, data from trials with monoclonal antibodies that target $B$ cells, such as Rituximab or Ocrelizumab showed that these medications may benefit even progressive MS and the theoretical explantation, could be related to effects on the B-cell mediated and compartmenized inflammation in the CNS [52-54]. Moreover, even when anti-CD20 monoclonal antibodies are injected intrathecally, they have a profound effect of peripheral B-cells [55], indicating that the BBB may be possibly passed easier from "inside-out" (ie from the CSF compartment to the blood). If indeed this is the case, then peripheral cleavage of antibodies by treatment modalities such as PLEX, may facilitate the efflux and clearance of the CNS antibodies, especially in patients with recent activity of MS and a compromised BBB.

The obvious drawback of our trial is that it is an open study without a control group. This was due to ethical issues imposed by the high activity of the disease and the fact that the patients did not respond to steroids and were not treated with any other immunomodulatory drug. The novelty of our trial is that PLEX was given as a chronic treatment (monthly courses following initial induction), for one year without the addition of other modalities, and that it showed significant clinical efficacy in active progressive MS. This selection of the patients (i.e., those with very active disease who were failures to steroids) may explain in part the more positive effects, detected in our trial as compared to previous ones. The recently revised criteria for the definition of progressive MS, which define the disease as either active or inactive, rather then progressive or relapsing $[57,58]$, may support the possibility that progressive disease can be influenced equally efficiently to RRMS, when it is active. It is possible that the cohort of patients in previous negative reports of PLEX in progressive MS, included cases with less active disease.

In line with this, our data show that the beneficial effects of PLEX were more pronounced in progressive MS patients with overimposed relapses (relapsing-progressive disease). The higher efficacy of PLEX in patients with a prominent myelitic presentation, and those with NMO, may indicate a common pathogenetic mechanism in these two subgroups, involving predominently humoral immunity. This comes in agreement with the subgroup analysis from previous studies [8] Additional, larger and controlled studies possibly involving progressive MS patients are warranted to confirrm our observations.

\section{References}

1. Clark WF, Rock GA, Buskard N, Shumak KH, LeBlond P, et al. (1999) Therapeutic plasma exchange: An update from the Canadian Apheresis Group. Ann Intern Med 131: 453-462.

2. Lehmann HC (2006) Plasma exchange in neuroimmunological disorders: Part 1: Rationale and treatment of inflammatory central nervous system disorders. Arch Neurol 63: 930-935.

3. Gordon PA (1985) A double-blind controlled pilot study of plasma exchange versus sham apheresis in chronic progressive multiple sclerosis. Can J Neuro Sci 12: 39-44.

4. Khatri BO, McQuillen MP, Harrington GJ, Schmoll D, Hoffmann RG (1985) Plasmapheresis in progressive MS. Neurology 35: 614

5. Weiner HL (1989) Double-blind study of true vs. sham plasma exchange in patients treated with immunosuppression for acute attacks of multiple sclerosis. Neurology 39: 1143-9.

6. (1991) The Canadian cooperative trial of cyclophosphamide and plasma 
exchange in progressive multiple sclerosis. The Canadian Cooperative Multiple Sclerosis Study Group. Lancet 337: 441-446.

7. Weinshenker BG (1999) A randomized trial of plasma exchange in acute central nervous system inflammatory demyelinating disease. Ann Neurol 46 : 878-886.

8. Keegan M (2005) Relation between humoral pathological changes in multiple sclerosis and response to therapeutic plasma exchange. Lancet 366: $579-582$

9. Lucchinetti C, Brück W, Parisi J, Scheithauer B, Rodriguez M, et al. (2000) Heterogeneity of multiple sclerosis lesions: Implications for the pathogenesis of demyelination. Ann Neurol 47: 707-717.

10. Magana SM (2011) Beneficial plasma exchange response in central nervous system inflammatory demyelination. Arch Neurol 68: 870-888.

11. Wang KC, Wang SJ, Lee CL, Chen SY, Tsai CP (2011) The rescue effect of plasma exchange for neuromyelitis optica. J Clin Neurosci 18: 43-46.

12. Watanabe S, Nakashima I, Miyazawa I, Misu T, Shiga Y, et al. (2007) Successful treatment of a hypothalamic lesion in neuromyelitis optica by plasma exchange. J Neurol 254: 670-671

13. Bonnan M, Valentino R, Olindo S, Mehdaoui H, Smadja D, et al. (2009) Plasma exchange in severe spinal attacks associated with neuromyelitis optica spectrum disorder. Mult Scler 15: 487-492.

14. Cortese I (2011) Evidence-based guideline update: Plasmapheresis in neurologic disorders: Report of the therapeutics and technology assessment subcommittee of the American Academy of Neurology. Neurology 76: 294-300

15. Sorensen PS (1996) Plasma exchange combined with azathioprine in multiple sclerosis using serial gadolinium-enhanced MRI to monitor disease activity: A randomized single-masked cross-over pilot study. Neurology 46:1620-1625.

16. Karussis D (2013) Immunotherapy of multiple sclerosis: the state of the art BioDrugs 27: 113-148.

17. Lehmann HC, Hartung HP, Hetzel GR, Stüve O, Kieseier BC (2006) Plasma exchange in neuroimmunological disorders: Part 2. Treatment of neuromuscular disorders. Arch Neurol 63: 1066-1071.

18. Lin CH, Jeng JS, Yip PK (2004) Plasmapheresis in acute disseminated encephalomyelitis. J Clin Apher 19: 154-159.

19. Miyazawa R, Hikima A, Takano Y, Arakawa H, Tomomasa T, et al. (2001) Plasmapheresis in fulminant acute disseminated encephalomyelitis. Brain Dev 23: $424-426$

20. Schröder A, Linker RA, Gold R (2009) Plasmapheresis for neurological disorders. Expert Rev Neurother 9: 1331-1339.

21. Faissner S, Nikolayczik J, Chan A, Hellwig K, Gold R, et al. (2016) Plasmapheresis and immunoadsorption in patients with steroid refractory multiple sclerosis relapses. J Neurol 263: 1092-1098.

22. Deschamps R, Gueguen A, Parquet N, Saheb S, Driss F, et al. (2016) Plasma exchange response in 34 patients with severe optic neuritis. J Neurol 263: 883-887.

23. Kleiter I, Gahlen A, Borisow N, Fischer K, Wernecke KD, et al. (2016) Neuromyelitis optica: Evaluation of 871 attacks and 1,153 treatment courses. Ann Neurol 79: 206-216.

24. Ehler J (2015) Response to Therapeutic plasma exchange as a rescue treatment in clinically isolated syndromes and acute worsening of multiple sclerosis: A retrospective analysis of 90 patients. PLoS One 10: e0134583.

25. Koziolek M (2013) Therapeutic apheresis in pediatric patients with acute CNS inflammatory demyelinating disease. Blood Purif 36: 92-97.

26. Meca-Lallana JE, Hernández-Clares R, León-Hernández A, Genovés Aleixandre A, Cacho Pérez M, et al. (2013) Plasma exchange for steroidrefractory relapses in multiple sclerosis: An observational, MRI pilot study. Clin Ther 35: 474-485.

27. Hawker K (2008) B-cell-targeted treatment for multiple sclerosis: Mechanism of action and clinical data. Curr Opin Neurol 21 Suppl 1: S19-25.

28. Zouali M (2008) B lymphocytes--chief players and therapeutic targets in autoimmune diseases. Front Biosci 13: 4852-4861.

29. Cepok S (2005) Short-lived plasma blasts are the main B cell effector subse during the course of multiple sclerosis. Brain 128:1667-1676.
30. Owens GP, Bennett JL, Gilden DH, Burgoon MP (2006) The B cell response in multiple sclerosis. Neurol Res 28: 236-244.

31. Prineas JW, Graham JS (1981) Multiple sclerosis: Capping of surface immunoglobulin $\mathrm{G}$ on macrophages engaged in myelin breakdown. Ann Neuro 10: $149-158$

32. Archelos JJ, Storch MK, Hartung HP (2000) The role of B cells and autoantibodies in multiple sclerosis. Ann Neurol 47: 694-706.

33. Serafini B, Rosicarelli B, Magliozzi R, Stigliano E, Aloisi F (2004) Detection of ectopic B-cell follicles with germinal centers in the meninges of patients with secondary progressive multiple sclerosis. Brain Pathol 14: 164-174.

34. Krumbholz M, Theil D, Cepok S, Hemmer B, Kivisäkk P, et al. (2006) Chemokines in multiple sclerosis: CXCL12 and CXCL13 up-regulation is differentially linked to CNS immune cell recruitment. Brain 129: 200-211.

35. Magliozzi R (2007) Meningeal B-cell follicles in secondary progressive multiple sclerosis associate with early onset of disease and severe cortical pathology. Brain 130: 1089-104.

36. Ruggieri S, Petracca M, Miller A, Krieger S, Ghassemi R, et al. (2015) Association of deep gray matter damage with cortical and spinal cord degeneration in primary progressive multiple sclerosis. JAMA Neurol 72: 14661474

37. Haider L (2014) Multiple sclerosis deep grey matter: the relation between demyelination, neurodegeneration, inflammation and iron. J Neurol Neurosurg Psychiatry 85: 1386-1395.

38. Calabrese M, Rinaldi F, Mattisi I, Bernardi V, Favaretto A, et al. (2011) The predictive value of gray matter atrophy in clinically isolated syndromes. Neurology 77: 257-263.

39. Cappellani R (2014) Subcortical deep gray matter pathology in patients with multiple sclerosis is associated with white matter lesion burden and atrophy but not with cortical atrophy: a diffusion tensor MRI study. AJNR Am J Neuroradiol 35: $912-919$

40. Ceccarelli A, Filippi M, Neema M, Arora A, Valsasina P, et al. (2009) T2 hypointensity in the deep gray matter of patients with benign multiple sclerosis. Mult Scler 15: 678-686.

41. Ceccarelli A, Rocca MA, Neema M, Martinelli V, Arora A, et al. (2010) Deep gray matter T2 hypointensity is present in patients with clinically isolated syndromes suggestive of multiple sclerosis. Mult Scler 16: 39-44.

42. Daams M, Geurts JJ, Barkhof F (2013) Cortical imaging in multiple sclerosis: Recent findings and 'grand challenges'. Curr Opin Neurol 26: 345-352.

43. Geurts JJ, Bö L, Pouwels PJ, Castelijns JA, Polman CH, et al. (2005) Cortical lesions in multiple sclerosis: Combined post-mortem MR imaging and histopathology. AJNR Am J Neuroradiol 26: 572-577.

44. Geurts JJ, Calabrese M, Fisher E, Rudick RA (2012) Measurement and clinica effect of grey matter pathology in multiple sclerosis. Lancet Neurol 11: 10821092

45. Neema M, Arora A, Healy BC, Guss ZD, Brass SD, et al. (2009) Deep gray matter involvement on brain MRI scans is associated with clinical progression in multiple sclerosis. J Neuroimaging 19: 3-8.

46. Kutzelnigg A, Faber-Rod JC, Bauer J, Lucchinetti CF, Sorensen PS, et al (2007) Widespread demyelination in the cerebellar cortex in multiple sclerosis. Brain Pathol 17: 38-44.

47. Kutzelnigg A, Lassmann $H$ (2006) Cortical demyelination in multiple sclerosis: A substrate for cognitive deficits? J Neurol Sci 245: 123-126.

48. Lucchinetti CF, Popescu BF, Bunyan RF, Moll NM, Roemer SF, et al. (2011) Inflammatory cortical demyelination in early multiple sclerosis. N Engl J Med 365: 2188-2197.

49. Popescu BF, Lucchinetti CF (2012) Meningeal and cortical grey matter pathology in multiple sclerosis. BMC Neurol 12: 11.

50. Romme Christensen J (2013) Systemic inflammation in progressive multiple sclerosis involves follicular T-helper, Th17- and activated B-cells and correlates with progression. PLoS One 8: e57820.

51. Petereit HF, A Rubbert-Roth (2009) Rituximab levels in cerebrospinal fluid of patients with neurological autoimmune disorders. Mult Scler 15: 189-192. 
Citation: Petrou P, Ben-Hur T, Vaknin-Dembinsky A, Abramsky O, Karussis D (2016) Clinical Efficacy of Plasma-Exchange in Patients with Progressive forms of Multiple Sclerosis and NMO-Spectrum Disease. J Mult Scler (Foster City) 3:181. doi:10.4172/2376-0389.1000181

52. Hawker K, et al. (2009) Rituximab in patients with primary progressive multiple sclerosis: results of a randomized double-blind placebo-controlled multicenter trial. Ann Neurol 66: 460-471.

53. Sorensen PS, M Blinkenberg (2016) The potential role for ocrelizumab in the treatment of multiple sclerosis: current evidence and future prospects. Ther Adv Neurol Disord 9: 44-52.

54. Kappos L, et al. (2011) Ocrelizumab in relapsing-remitting multiple sclerosis: a phase 2, randomised, placebo-controlled, multicentre trial. Lancet 378: 17791787.
55. Studer V, et al. (2014) Peripheral B cell depletion and central proinflammatory cytokine reduction following repeated intrathecal administration of rituximab in progressive Multiple Sclerosis. J Neuroimmunol 276: 229-231.

56. Komori M, et al. (2016) Insufficient disease inhibition by intrathecal rituximab in progressive multiple sclerosis. Ann Clin Transl Neurol 3: 166-179.

57. Lublin FD, et al. (2014) Defining the clinical course of multiple sclerosis: the 2013 revisions. Neurology 83: 278-286.

58. Lublin FD, et al. (2014) New multiple sclerosis phenotypic classification. Eur Neurol 72: 1-5
OMICS International: Publication Benefits \& Features

Unique features:

- Increased global visibility of articles through worldwide distribution and indexing

- Showcasing recent research output in a timely and updated manner

Special issues on the current trends of scientific research

Special features:

- $700+$ Open Access Journats

$50,000+$ editorial team

Rapid review process

- Quality and quick editorial, review and publication processing

- Indexing at major indexing services

- Sharing Option: Social Networking Enabled

- Authors, Reviewers and Editors rewarded with online Scientific Credits

- Befter discount for your subsequent articles

Submit your manuscript at: http://www.omicsonline.org/submission/ 